\title{
A novel combined ex vivo and in vivo lentiviral interleukin-10 gene delivery strategy at the time of transplantation decreases chronic lung allograft rejection in mice
}

\author{
Hisashi Oishi, MD, PhD, ${ }^{a}$ Stephen C. Juvet, MD, PhD, ${ }^{a}$ Tereza Martinu, MD, ${ }^{\text {a }}$ Masaaki Sato, MD, PhD, ${ }^{\mathrm{a}, \mathrm{b}}$
}

Jeffrey A. Medin, PhD, ${ }^{\mathrm{c}}$ Mingyao Liu, MD, MSc, ${ }^{\mathrm{a}}$ and Shaf Keshavjee, MD, MSc ${ }^{\mathrm{a}}$

\section{ABSTRACT}

Objective: Our objective was to develop a rapid-onset and durable gene-delivery strategy that is applicable at the time of transplant to determine its effects on both acute rejection and chronic lung allograft fibrosis using a mouse orthotopic lung transplant model.

Methods: C57BL/6 mice received an orthotopic left lung transplant from syngeneic donors or C57BL/10 donors. By using syngeneic lung transplantation, we established a novel gene transfer protocol with lentivirus luciferase intrabronchial administration to the donor lung ex vivo before transplantation. This strategy was applied in allogeneic lung transplantation with lentivirus engineering expression of human interleukin-10 or lentivirus luciferase (control).

Results: Bioluminescent imaging revealed that the highest early transgene expression was achieved when lentivirus luciferase was administered both directly into the donor lung graft ex vivo before implantation and subsequently to the recipient in vivo daily on post-transplant days 1 to 4 , compared with post-transplant in vivo administration only (days 0 to 4). Our previous work with adenoviral interleukin-10 gene therapy indicates that early interleukin-10 expression in the allograft is desirable. Therefore, we selected the combined protocol for human interleukin-10 encoding lentiviral vector therapy. In the allogeneic transplant setting, ex vivo and in vivo human interleukin-10 encoding lentiviral vector therapy reduced acute rejection grade $(2.0$ vs $3.0, P<.05)$ at day 28. The percentage of fibrotic obliterated airways was reduced in the human interleukin-10 encoding lentiviral vector-treated group $(10.9 \% \pm 7.7 \%$ vs $40.9 \% \pm 9.3 \%, P<.05)$.

Conclusions: Delivery of lentiviral interleukin-10 gene therapy, using a novel combined ex vivo and in vivo delivery strategy, significantly improves acute and chronic rejection in the mouse lung transplant model. ( $\mathrm{J}$ Thorac Cardiovasc Surg 2018;156:1305-15)

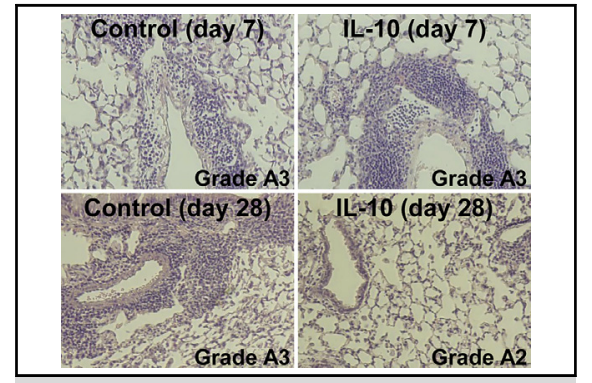

Representative histology pictures of each group at days 7 and 28 .

\section{Central Message}

Lentiviral IL-10 gene therapy, using a novel combined ex vivo and in vivo delivery strategy, significantly improves acute and chronic rejection in a mouse lung transplant model.

\section{Perspective}

Lentiviral hIL-10 gene therapy delivered at the time of lung transplant in a combined ex vivo and in vivo technique provides rapid and durable upregulation of IL-10 expression that inhibits acute and chronic rejection in a mouse lung transplant model. These beneficial effects were accompanied by upregulation of endogenous IL-10 and LAG-3, suggesting the involvement of $\operatorname{Tr} 1$ regulatory cells.

See Editorial Commentary page 1316.

See Editorial page 1303.

\footnotetext{
From the ${ }^{\mathrm{a}}$ Latner Thoracic Surgery Research Laboratories, University Health Network, University of Toronto, Toronto, Ontario, Canada; ${ }^{b}$ Department of Thoracic Surgery, University of Tokyo, Tokyo, Japan; and ${ }^{\mathrm{c}}$ Departments of Pediatrics and Biochemistry, Medical College of Wisconsin, Milwaukee, Wis.

This research was supported by the Canadian Institutes of Health Research and the Canadian Cystic Fibrosis Foundation.

Received for publication Dec 12, 2017; revisions received April 23, 2018; accepted for publication May 1, 2018; available ahead of print June 21, 2018.

Address for reprints: Shaf Keshavjee, MD, MSc, Toronto General Hospital, 200 Elizabeth St, 9N-946, Toronto M5G 2C4, Ontario, Canada (E-mail: shaf.keshavjee@ uhn.ca).

0022-5223/\$36.00

Copyright (C) 2018 Published by Elsevier Inc. on behalf of The American Association for Thoracic Surgery

https://doi.org/10.1016/j.jtcvs.2018.05.003
}

Lung transplantation remains the only therapeutic option for patients with end-stage lung disease. Although acute rejection (AR) is not a leading cause of death after lung transplantation ${ }^{1}$ because of immunosuppressive agents, administration of these drugs causes serious complications. In

- Scanning this $\mathrm{QR}$ code will take you to a supplemental video for the article. 


\section{Abbreviations and Acronyms \\ $\mathrm{AR}=$ acute rejection \\ CLAD = chronic lung allograft dysfunction \\ ELISA = enzyme-linked immunosorbent assay \\ EVLP = ex vivo lung perfusion \\ Foxp3 = forkhead box P3 \\ hIL-10 = human interleukin-10 \\ IL-10 = interleukin-10 \\ LAG-3 = lymphocyte-activation gene 3 \\ LV-hIL-10 = human interleukin-10 encoding lentiviral vector \\ LV-Luc = luciferase-encoding lentiviral vector \\ PGD = primary graft dysfunction \\ $\operatorname{Tr} 1=$ T-regulatory type 1}

contrast, chronic lung allograft dysfunction (CLAD) is the most significant cause of death 1 year post-transplant, and unfortunately it is still not preventable with the use of modern immunosuppression. CLAD is generally thought to represent chronic rejection and manifests most commonly as obliterative bronchiolitis, with approximately $30 \%$ of patients also exhibiting parenchymal fibrosis.

Lung graft inflammation resulting from donor brain death or ischemia-reperfusion injury can lead to primary graft dysfunction (PGD). PGD is linked to a higher risk for $\mathrm{CLAD}^{2}$; therefore, it stands to reason that control of early inflammatory insults may help to prevent CLAD development. Pretransplant anti-inflammatory or immune regulatory treatments are an attractive option to pretreat the graft before exposure to the host immune system at implantation. Ex vivo delivery is also attractive in that the treatment can be administered to the lung graft without affecting other organs in the donor.

In line with this hypothesis, we have shown that adenovirusmediated human interleukin-10 (hIL-10) gene therapy ameliorates ischemia-reperfusion injury and improves lung graft function in $\mathrm{rat}^{3-5}$ and $\mathrm{pig}^{6}$ lung transplant models. More recently, we reported that the ex vivo adenoviral hIL-10 gene delivery to the graft during normothermic ex vivo lung perfusion (EVLP) improved early lung allograft function in a pig acute lung transplant model ${ }^{7,8}$ and a pig survival lung transplant model. ${ }^{9}$ In addition, we have demonstrated that adenoviral hIL-10 gene transfer during ex vivo perfusion of human donor lungs rejected for transplantation resulted in physiologic and structural improvement during EVLP.

A limitation of adenoviral gene therapy is short-term gene expression, ${ }^{10}$ which may be suitable only for the treatment and modulation of early post-transplant inflammation. In contrast, lentivirus vectors can provide longer-term gene expression. We previously demonstrated that lentiviral hIL10 delivered through the airway achieved transgene expression in the mouse lung for more than 1 year $^{11}$ and attenuated
AR in a mouse orthotopic lung transplant model. ${ }^{12}$ In that previous proof-of-principle study, gene transfer was performed to both donor and recipient; however, in vivo administration of therapeutic vectors to human lung allograft donors is clinically impractical. Furthermore, the effects of hIL-10 gene therapy on development of longer-term chronic rejection were not examined. Shoji and colleagues ${ }^{13}$ have demonstrated that post-transplant delivery of the mouse interleukin-10 (IL-10) gene using a recombinant Sendai virus vector led to reduced luminal obliteration in a heterotopic tracheal transplant model, suggesting that IL-10 gene therapy may inhibit chronic rejection; however, the nonvascularized model of that study lacks classic ischemia-reperfusion injury and some of the relevant features of human lung transplantation. ${ }^{13}$

We hypothesized that lentiviral hIL-10 gene therapy administered ex vivo and in vivo would achieve early transgene expression and ameliorate subsequent acute and chronic rejection in a minor-mismatched orthotopic mouse single lung transplant model.

\section{MATERIALS AND METHODS \\ Mouse Orthotopic Lung Transplant}

Male 8-to-12-week-old C57BL/6J and C57BL/10J mice were purchased from Jackson Laboratories (Bar Harbor, Me). Mouse orthotopic left lung transplantation (Video 1) was performed according to Okazaki and colleagues. ${ }^{14}$ All animals received care in compliance with the Guide to the Care and Use of Experimental Animals formulated by the Canadian Council on Animal Care. The experimental protocol was approved by the Animal Care Committee of the Toronto General Hospital Research Institute, University Health Network.

\section{Ex Vivo Gene Transfer to Donor Graft Pretransplantation}

The heart-lung block was extracted as previously described. ${ }^{11}$ Left hilar structures were dissected, and the pulmonary artery and vein were cuffed.

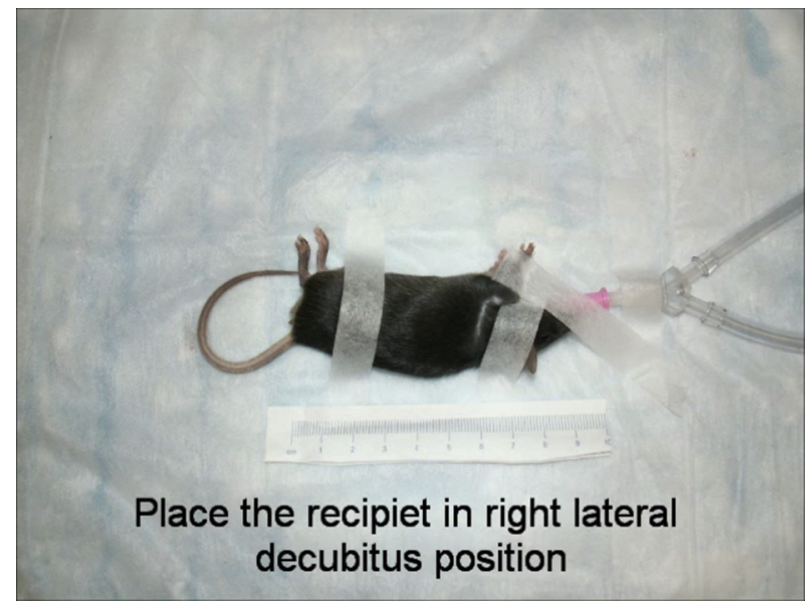

VIDEO 1. Mouse orthotopic lung transplant technique. This video shows the mouse orthotopic lung transplant technique as performed at our laboratory. Video available at: https://www.jtcvs.org/article/S0022-5223(18) 31419-3/fulltext. 

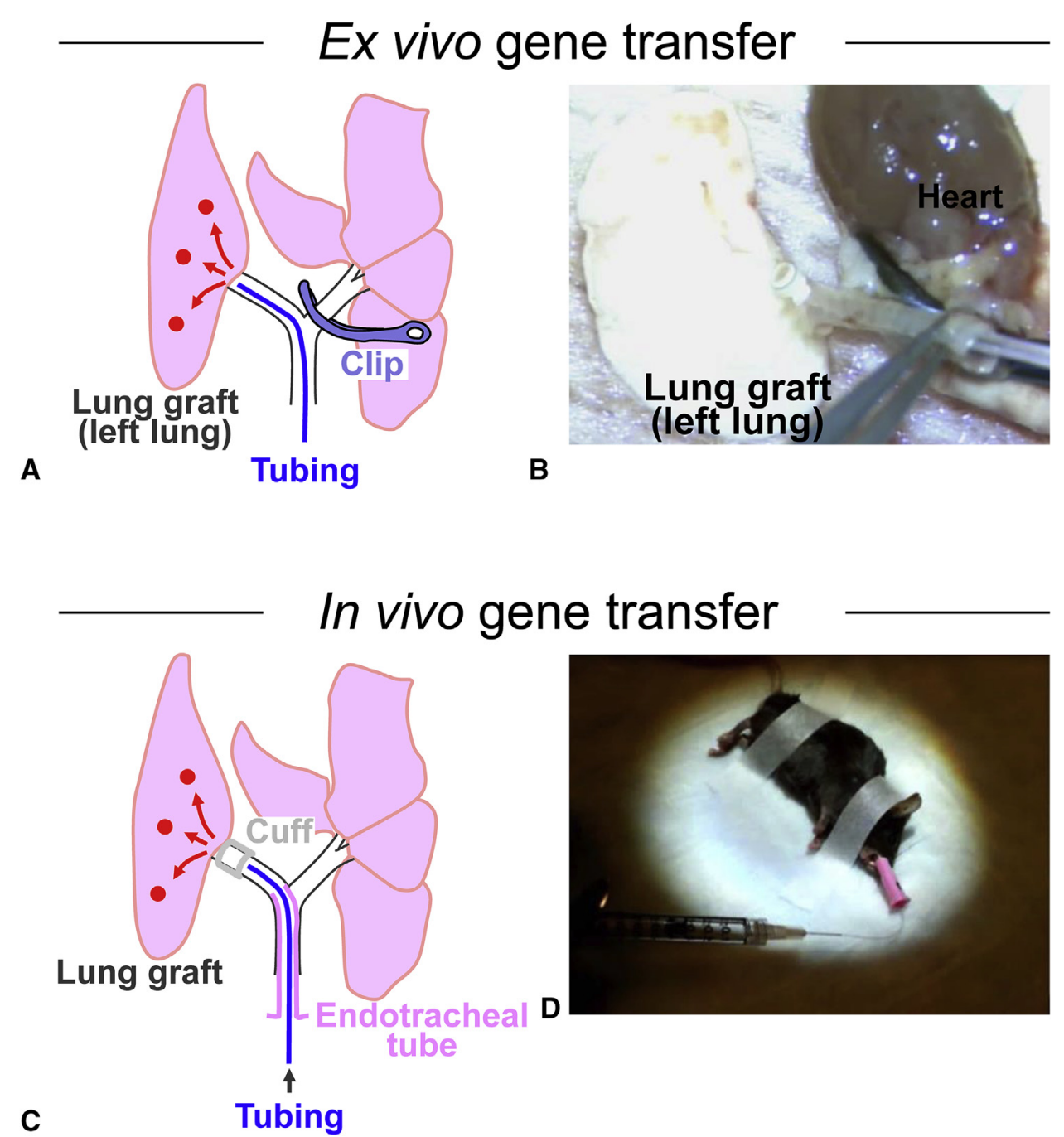

FIGURE 1. Two gene transfer techniques used in this study: ex vivo and in vivo gene transfer. A, Schematic picture of ex vivo gene transfer. B, For ex vivo gene transfer, the heart-lung block was placed on cooled saline-soaked gauze. A micro-tube was inserted into the left main stem bronchus through the trachea, and lentiviral vectors were delivered transbronchially to the left lung. C, Schematic picture of in vivo gene transfer. D, For in vivo gene transfer, the recipient mouse was anesthetized, intubated with a 20-gauge peripheral venous catheter, and connected to a small-animal ventilator, and then put in the left lateral decubitus position. The intubation catheter was bent toward the left bronchus, and we confirmed its location in the left bronchus by observing left chest wall respiratory movement in the absence of right chest wall movement. Lentiviral vectors were delivered to the graft via a micro-tube inserted through the intubation catheter.

Before placing the bronchial cuff, luciferase-encoding lentiviral vector (LVLuc) or human IL-10 encoding lentiviral vector (LV-hIL-10) was delivered intrabronchially through 10-gauge polyethylene tubing (PE10; Becton Dickinson Canada, Mississauga, Canada) (Figure 1, $A$ and $B$; Video 2). Each dose contained similar p24 titers of LV-Luc or LV-hIL-10 in $40 \mu \mathrm{L}$ of phosphatebuffered saline plus $100 \mu \mathrm{L}$ of air. The lentivirus-treated lung graft was then transplanted to the recipient as previously described. ${ }^{14}$

\section{In Vivo Gene Transfer After Lung Transplant}

After recipient anesthesia, endotracheal intubation was performed using a 20-gauge venous catheter (BD Angiocath; Becton Dickinson, Mississauga, Ontario, Canada), and the mouse was connected to a small-animal ventilator (Model 693, Harvard Apparatus, Holliston, Mass) and placed in left lateral decubitus position. The catheter was bent toward the left bronchus; preferential left lung ventilation was confirmed by observing left chest wall movement. PE10 tubing was inserted through the catheter, and LV-Luc or LV-hIL-10 was delivered to the lung graft in the same dose as for ex vivo gene transfer (Figure 1, $C$ and $D$; Video 3).

\section{Assessment of Gene Transfection With In Vivo Bioluminescent Imaging and Enzyme-Linked Immunosorbent Assay}

LV-Luc and LV-hIL-10 were produced as described previously. ${ }^{12}$ For bioluminescent imaging, LV-Luc-treated mice were given D-Luciferin (150 mg/kg intraperitoneally; Cayman Chemical, Ann Arbor, Mich) and imaged using a Xenogen IVIS Imaging System 100 (Caliper Life Sciences, Waltham, Mass) under light isoflurane anesthesia. Expression of hIL-10 in the lung graft, lung tissue homogenization, and protein extraction were performed as described. ${ }^{15}$ Serum and lung tissue homogenates were assayed 


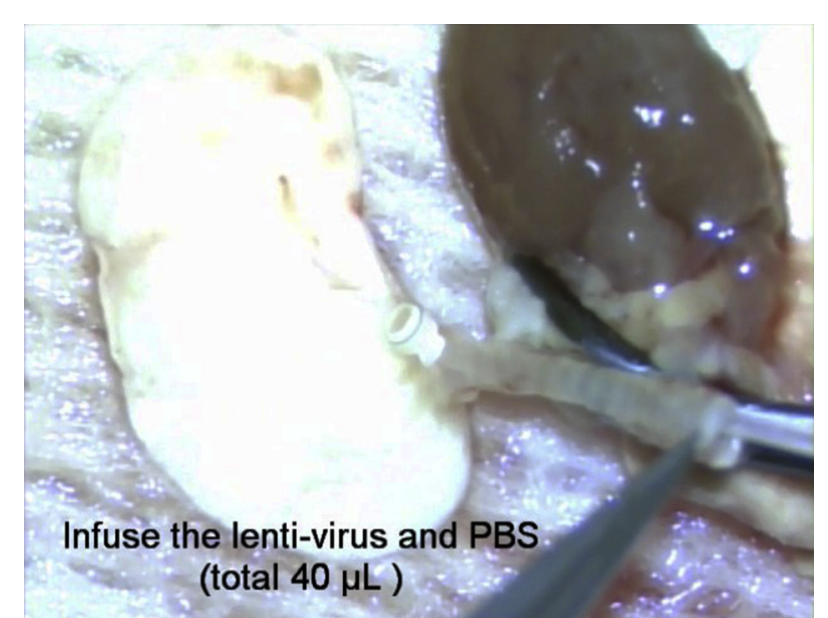

VIDEO 2. Ex vivo gene transfer to donor graft pretransplantation. The heart-lung block was placed on cooled saline-soaked gauze. A micro-tube was inserted into the left main stem bronchus through the trachea, and lentiviral vectors were delivered transbronchially to the left lung. Video available at: https://www.jtcvs.org/article/S0022-5223(18)31419-3/fulltext.

with an enzyme-linked immunosorbent assay (ELISA) kit (IL-10 Human Ultrasensitive ELISA Kit; Invitrogen, Carlsbad, Calif). Total protein concentration, determined as described, ${ }^{15}$ was used as the denominator.

\section{Grading of Rejection, Airway Obliteration, and Lung Parenchymal Fibrosis}

AR was graded using the International Society for Heart and Lung Transplantation criteria. ${ }^{16}$ The percentages of obliterated airways and lung parenchyma affected by parenchymal fibrosis were determined. All pathology grading was performed by an operator experienced in rodent lung pathology assessment and blinded to group assignment.

\section{Immunofluorescent Staining for Foxp3}

Immunofluorescent staining for forkhead box P3 (Foxp3) was conducted as described previously. ${ }^{17}$ Images were acquired using a spinning disk confocal system (WaveFX; Yokogawa Electric Corp, Tokyo, Japan). Ten high-power images were randomly acquired from 3 sections per animal, and the number of Foxp $3^{+}$cells was counted in a blinded manner.

\section{Statistical Analysis}

Data are expressed as means \pm standard error of the mean. A nonparametric Mann-Whitney $U$ test was performed for comparison of the means and AR grades of 2 groups. Student $t$ test or Welch's $t$ test was used for parametric data. Linear regression was used to determine correlations. Statistical analyses were performed using Prism 5 (GraphPad Software Inc, La Jolla, Calif). For luciferase activity, a linear mixed-effects model and post hoc Welch's $t$ test were performed using $\mathrm{R}$ version 3.1.0 (lme4 package version 1.1-6, The R Foundation for Statistical Computing, Vienna, Austria).

\section{RESULTS}

\section{Lentivirus-Mediated Gene Transfer Using Combined Ex Vivo and In Vivo Delivery Enables Effective Early and Long-Term Transgene Expression in a Syngeneic Mouse Lung Transplant Model}

To assess gene transfer efficiency in potentially clinically applicable models, syngeneic mouse orthotopic left lung

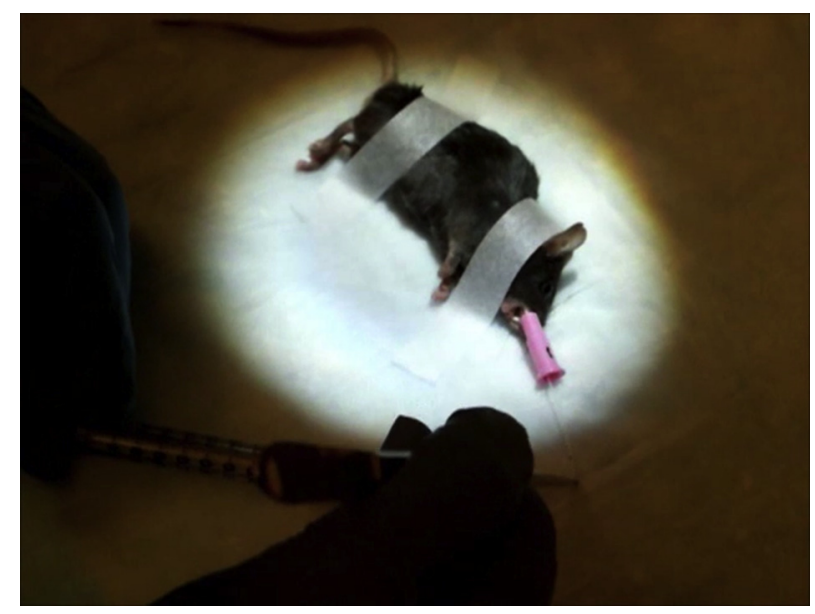

VIDEO 3. In vivo gene transfer after lung transplant. The recipient mouse was anesthetized, intubated with a 20-gauge peripheral venous catheter, connected to a small-animal ventilator, and put in the left lateral decubitus position. The intubation catheter was bent toward the left bronchus. Lentiviral vectors were delivered to the graft via a micro-tube inserted through the intubation catheter. Video available at: https://www.jtcvs.org/article/ S0022-5223(18)31419-3/fulltext.

transplants were performed using C57BL/6 mice as donors and recipients. We first performed in vivo gene transfer to recipients using LV-Luc on post-transplant days 1 to 4 (Figure 2, A) and measured luciferase expression in the transplanted lung using in vivo bioluminescent imaging (Figure 2, B). Expression levels in the acute and chronic phases were relatively low, compared with previous work in which donor lungs received LV-Luc 7 days before lung transplant (Figure 2, $C, \mathrm{n}=4$ ) ${ }^{11,12}$ In addition, because we did not deliver LV-Luc at day 0 , gene expression was not detected on day 1 . To evaluate strategies to overcome these limitations, we studied 2 additional groups of mice. In the first group, LV-Luc was administered to the allograft ex vivo at the time of retrieval and subsequently to the recipients in vivo after transplant (ex vivo and in vivo gene transfer, $\mathrm{n}=6$ ) (Figure 3, $A$ ). In the second group, LV-Luc was administered to recipient mice after lung transplantation from days 0 to 4 (in vivo only gene transfer, $n=6$ ) (Figure 3, A). In this latter group, day 0 administration was performed immediately after lung transplantation. Both groups showed stable long-term gene expression, and expression levels in the chronic phase were similar (Figure 3, B). However, gene expression levels in the ex vivo and in vivo gene transfer group at days 1 and 2 were higher than in the in vivo only gene transfer group (Figure 3,C).

By using adenoviral vectors to deliver IL-10 gene therapy, we have previously shown that immediate human IL10 expression in the transplanted lung can ameliorate ischemia-reperfusion injury, ${ }^{3}$ inflammatory cytokine production, ${ }^{4}$ and necrotic cell death. ${ }^{5}$ Further, adenoviral IL10 gene therapy facilitated functional repair of damaged 


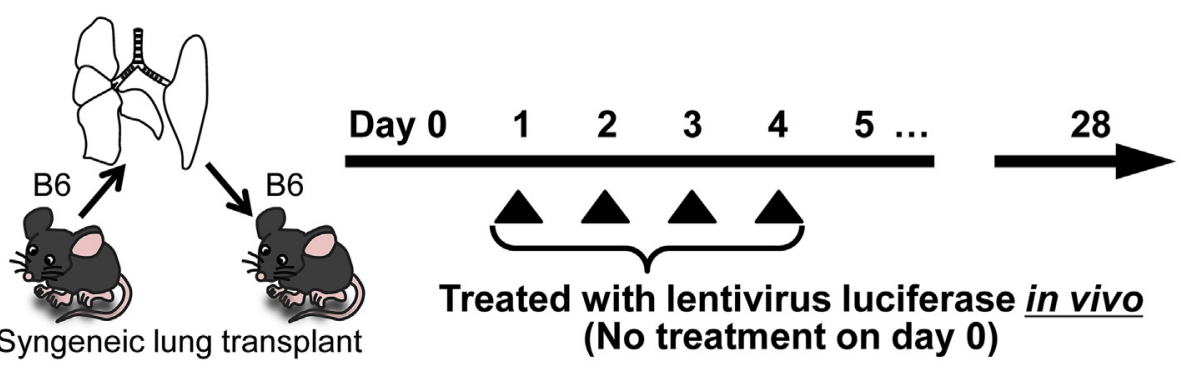

B
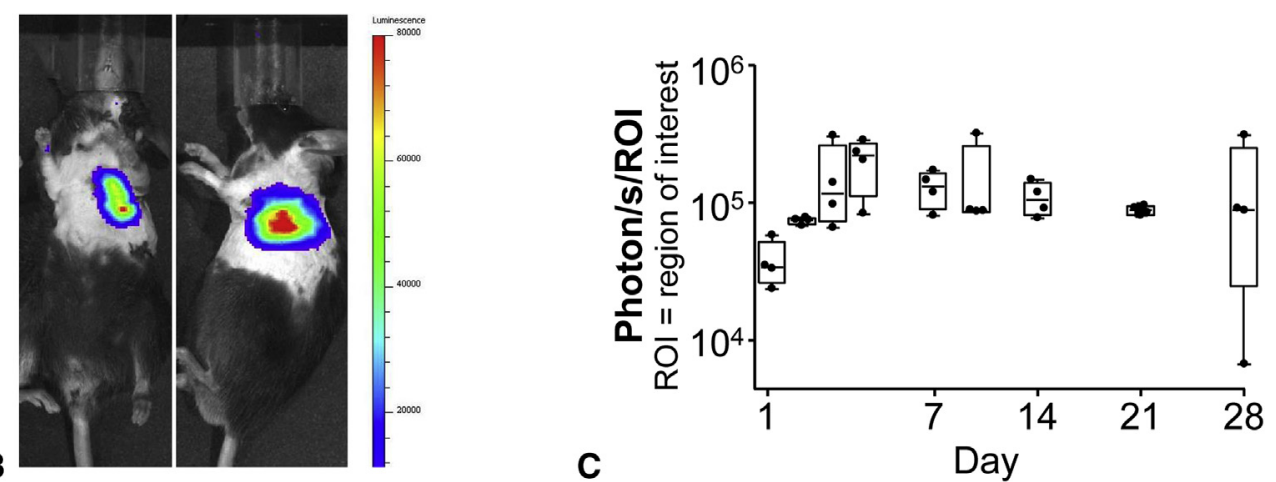

FIGURE 2. Transgene expression after lung transplantation and in vivo gene delivery. C57BL/6 mice received left lung isografts from C57BL/6 donors and were treated with LV-Luc. A, Gene transfer was performed in vivo after lung transplant on days 1 to 4 . B, Bioluminescent imaging of a representative mouse treated with LV-Luc at post-transplant day 4. Bioluminescence is limited to the transplanted lung. C, Post-transplant gene expression levels over time as measured by luciferase activity. The upper and lower borders of the box represent the upper and lower quartiles. The middle horizontal line represents the median. The upper and lower borders of the box represent the upper and lower quartiles. The middle horizontal line represents the median. The upper and lower whiskers represent the maximum and minimum values of nonoutliers. Extra dots represent outliers. Data are from n $=4$ animals. ROI, Region of interest.

human lungs during EVLP. ${ }^{7}$ In the context of this body of evidence and the data shown in Figure 3, we therefore selected the combined ex vivo and in vivo gene transfer method for further experiments.

\section{Lentivirus-Mediated Human Interleukin-10}

Transgene Delivery Using Combined Ex Vivo and In Vivo Gene Transfer Achieves Effective Early and Long-Term Human Interleukin-10 Protein Expression in a Minor Mismatch Mouse Lung

\section{Transplant Model}

We next investigated the kinetics of hIL-10 expression using the combined ex vivo and in vivo gene transfer approach in the minor alloantigen-mismatch lung transplant model. We determined hIL-10 protein expression level at days 1,7 , and 28 post-transplant by ELISA (Figure 4, $A$ ) $(\mathrm{n}=4$ for day 1 group, $\mathrm{n}=4$ for day 7 group, and $\mathrm{n}=5$ for day 28 group). Graft hIL-10 was detectable at days 1 , 7 , and 28 post-transplant by ELISA (Figure 4, B). Expression was maintained at day $28(13.6 \pm 2.5 \mathrm{pg} / \mathrm{mg}$ protein $)$.

\section{Lentivirus-Mediated Human Interleukin-10}

\section{Transgene Delivery Using Combined Ex Vivo and} In Vivo Gene Transfer Ameliorates Acute Rejection

Blinded pathologic scoring according to the International Society for Heart and Lung Transplantation criteria demonstrated no significant difference in AR A grades between the 2 groups at day 7 post-transplant (median 3.0 vs 3.0; Figure 5, $A-C$; control group, $\mathrm{n}=8$; IL-10 group, $\mathrm{n}=7$ ). At day 28 post-transplant, however, there were fewer infiltrating cells in the IL-10 group and the A grade was significantly lower compared with the control group (median 2.0 vs 3.0; Figure 5, D-F; $P<.05$; control group, $\mathrm{n}=11 ;$ IL-10 group, $\mathrm{n}=13 ; 1$ lung graft from the control group could not be scored because of the severity of the fibrosis).

\section{Lentivirus-Mediated Human Interleukin-10}

Transgene Delivery Using Ex Vivo and In Vivo Gene Transfer Decreases Airway Obliteration and Lung Allograft Fibrosis

We examined day 28 histologic sections for evidence of allograft fibrosis and airway obliteration, both pathologic features of human CLAD, ${ }^{18}$ in response to combined ex vivo and in vivo LV-hIL-10 gene transfer. In the control group, airway obliteration with fibrous tissue was seen in the majority of mice (8/12; Figure 5, $G$ and $I$ ). In the IL10 group, airway obliteration was observed in only 2 of 13 mice $(P<.05$, Figure $5, H$ and $I)$. The percentage of fibrotic obliterated airways, a feature of chronic rejection, was significantly reduced in the LV-hIL-10-treated group $(10.9 \% \pm 7.7 \%$ vs $40.9 \% \pm 9.3 \%, P<.05$; Figure $5, I)$. 

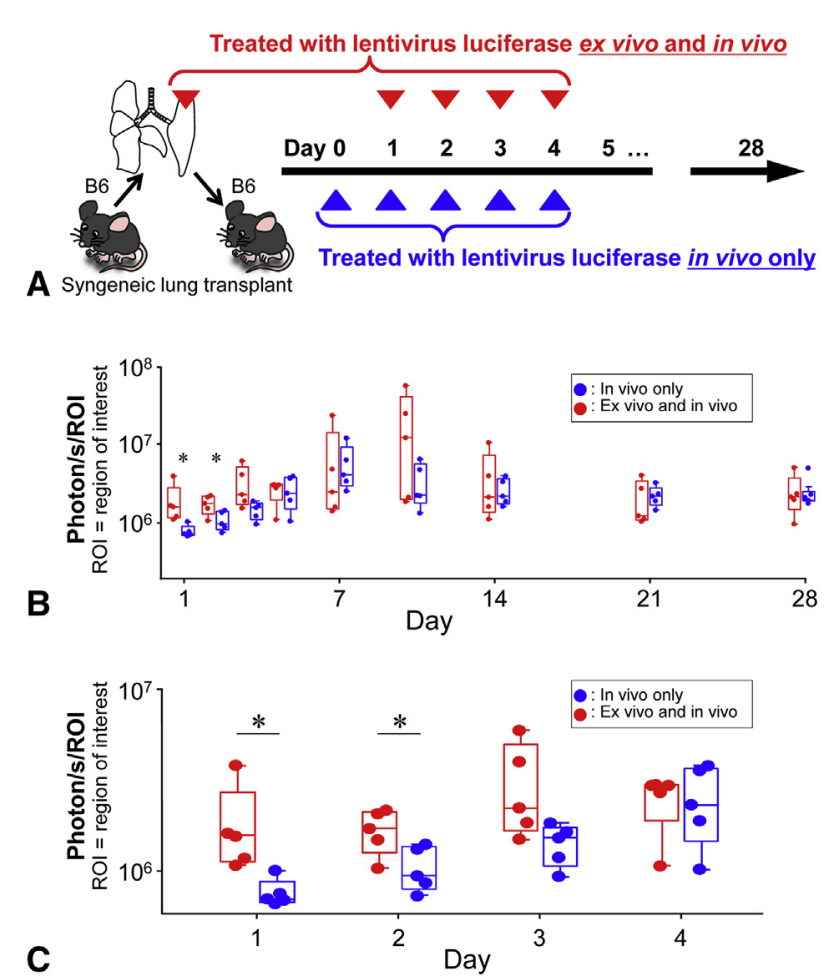

FIGURE 3. Combined ex vivo and in vivo gene transfer enables effective early and long-term transgene expression. Isografts were treated with LVLuc ex vivo and in vivo or in vivo only. A, Treatment scheme for the 2 groups. In vivo treatments were given on days 0 to 4 (in vivo only group, red triangles) or days 1 to 4 (combined ex vivo and in vivo group, blue triangles; same total number of treatments in both groups). B, Both groups showed stable gene expression over 28 days. C, At days 1 and 2 posttransplant, gene expression was higher in the combined ex vivo and in vivo treatment group than in the in vivo only treatment group. The middle horizontal line represents the median. Extra dots represent outliers. Data are from $\mathrm{n}=6$ animals. *Linear mixed-effects model and post hoc Welch's $t$ test $P<.05$. ROI, Region of interest.

Parenchymal fibrotic changes tended to be more severe in the control group than in the IL-10 treatment group (Figure 5, $J$ and $K$ ). Although the difference between the groups did not reach statistical significance, only 2 of 13 mice $(15 \%)$ in the IL-10 group had severe fibrotic changes compared with 5 of 12 mice $(42 \%)$ in the control group (Figure $5, L$ ). We next examined lung gene expression using quantitative real-time polymerase chain reaction. Expression of collagen type 1 alpha 1 in the allograft was higher in the control group than in the IL-10 group at day 28 $(P<.05$, Figure $5, M$; control group, $\mathrm{n}=12$; IL-10 group, $\mathrm{n}=13$ ). Stable hIL-10 human gene expression was detected in all lung grafts in the IL-10 group at days 7 and 28 posttransplant (Figure 5, $\mathrm{N}$; control group at days 7 and 28, $\mathrm{n}=8$ and 12 ; IL-10 group at days 7 and $28, \mathrm{n}=7$ and 13). These findings show that administration of lentiviral hIL-10 gene therapy in a combined ex vivo and in vivo fashion leads to reduced AR and diminished features of chronic rejection.

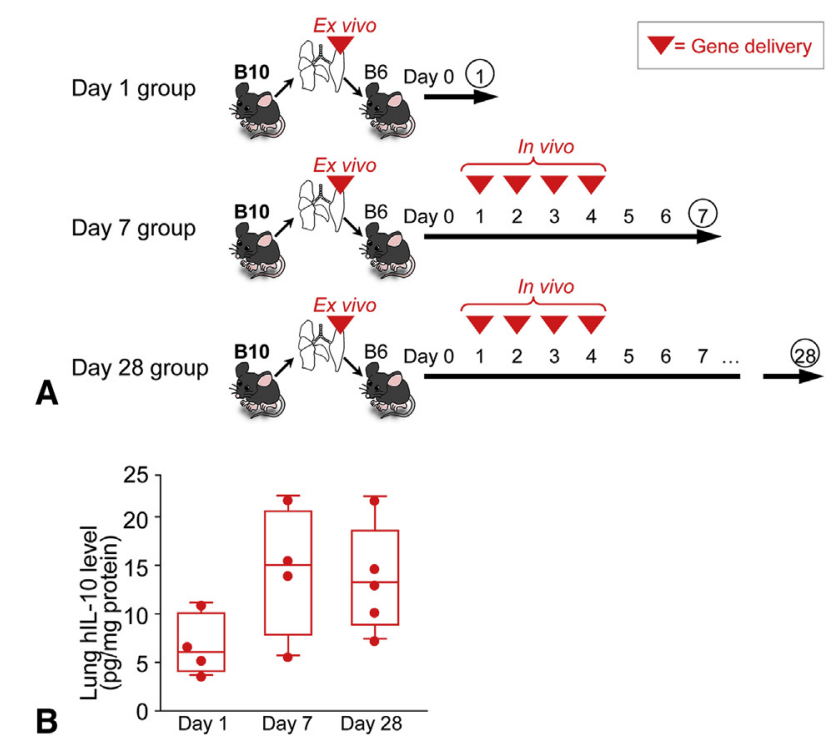

FIGURE 4. Lentivirus-mediated human IL-10 gene delivery achieves effective early and late human IL-10 expression in the lung allograft. A, In the day 1 group, LV-hIL-10 was administered to the lung graft by ex vivo gene transfer, and recipients were killed at day 1 post-transplant. In the day 7 group, LV-hIL-10 gene transfer was performed ex vivo and in vivo after lung transplant daily on days 1 to 4 ; recipient mice were sacrificed at day 7 . Mice in the day 28 group were treated as the day 7 group but killed at day 28. B, Human IL-10 levels in the lung graft were measured by a human-specific ELISA. In the day 1 group, hIL-10 level in the graft was $6.7 \pm 1.6 \mathrm{pg} / \mathrm{mg}$ protein. In the day 7 group, the expression level reached a peak of $14.5 \pm 3.4 \mathrm{pg} / \mathrm{mg}$ protein. Gene expression level was maintained until day 28 (13.6 $\pm 2.5 \mathrm{pg} / \mathrm{mg}$ protein). The middle horizontal line represents the median. The upper and lower borders of the box represent the upper and lower quartiles. The middle horizontal line represents the median. The upper and lower whiskers represent the maximum and minimum values of nonoutliers. Data are from $\mathrm{n}=4$ for day 1 group, $\mathrm{n}=4$ for day 7 group, and $\mathrm{n}=5$ for day 28 group. hIL-10, Human interleukin-10.

The data shown in Figure 5 reveal that compared with the control group, the IL-10-treated group experienced decreased features of AR and chronic rejection at day 28 . At day 7, however, AR scores were similar. This observation led us to speculate that the composition, rather than abundance, of cellular infiltrates in IL-10-treated and control allografts at this stage may differ. Specifically, we hypothesized that IL-10 treatment may have promoted the influx of regulatory cells into the allograft. IL-10 is a product of regulatory T and B lymphocytes, ${ }^{19,20}$ and so we tested whether hIL-10 resulted in the expression of endogenous mouse-derived mIL-10 in lung allografts. As shown in Figure 6, A, mouse-derived IL-10 (mIL-10) transcripts were expressed at similar levels at day 7 in the control and IL-10 groups. By day 28, however, mIL-10 expression was approximately 3 -fold higher in the IL-10 group compared with the control group $(P=.02$; control group at days 7 and 28, $\mathrm{n}=8$ and 12; IL-10 group at days 7 and $28, \mathrm{n}=7$ and 13). The induction of mIL-10 transcripts in 
the allograft correlated with the level of hIL-10 expression $\left(\mathrm{R}^{2}=0.43, P<.05\right.$; control group, $\mathrm{n}=12$; $\mathrm{IL}-10$ group, $\mathrm{n}=13$ ), suggesting that exogenous IL-10 drives further endogenous IL-10 expression in the graft. CD4 ${ }^{+}$Foxp $^{+}$ Tregs can secrete IL-10 and inhibit allograft rejection. ${ }^{18}$

The ratio of Foxp3 to CD4 and the total number of Foxp $^{+}$nuclei in lung allografts were similar between IL-10 and control groups at day 28 (Figure 6, C-F; control group, $\mathrm{n}=5$; IL-10 group, $\mathrm{n}=5$ ). These data suggest that the increased mIL-10 seen in the IL-10 group was not likely due to a difference in Foxp $3^{+}$Treg infiltration. $\mathrm{CD}^{+}{ }^{+}$T-regulatory type 1 ( $\left.\operatorname{Tr} 1\right)$ cells, which also secrete IL-10, can naturally regulate immune responses and induce tolerance to alloantigens in vivo in solid-organ transplantation. ${ }^{21} \mathrm{CD} 49 \mathrm{~b}$ and lymphocyte-activation gene 3 (LAG-3) are $\operatorname{Tr} 1$ cell markers. We were unable to demonstrate LAG-3 or CD49b expression by immunofluorescent staining of allograft sections, so we elected to examine their transcripts in IL-10-treated and control allografts. The ratio of CD49b to CD4 expression was similar at days 7 and 28 in the control and IL-10 groups (Figure 7, $A$; control group at days 7 and 28, $\mathrm{n}=8$ and 12 ; IL-10 group at days 7 and 28, $\mathrm{n}=7$ and 13). However, the ratio of LAG-3 to CD4 expression was 2-fold higher in the IL-10 group than in the control group at day 28 (Figure 7, $B, P<.01$; control group at days 7 and $28, \mathrm{n}=8$ and 12 ; IL-10 group at days 7 and 28, $\mathrm{n}=7$ and 13). CD49b is an integrin that is also expressed on natural killer cells and natural killer T cells, ${ }^{22}$ as well as on fibroblasts. ${ }^{23}$ In a CLAD model characterized by inflammatory and fibrotic change, its expression may be less specific for $\operatorname{Tr} 1$ cells. In contrast, LAG-3 expression is consistently associated with immune regulation in a number of settings. ${ }^{24}$ In keeping with this notion, we observed a close relationship between mIL-10 expression and LAG-3 expression in lung allografts $\left(\mathrm{R}^{2}=0.89, P<.0001\right.$, Figure $7, C ;$ control group, $\mathrm{n}=12$; IL-10 group, $\mathrm{n}=13$ ). Our findings suggest that combined IL-10 gene therapy delivered ex vivo and in vivo may promote the induction of $\operatorname{Tr} 1$ cells within the allograft.

\section{DISCUSSION}

We have shown that combined ex vivo and in vivo lentivirus-mediated gene transfer enables immediate posttransplant and long-term gene expression of the transgene product. We further demonstrated that lentivirus-mediated hIL-10 gene transfer, using this novel strategy, significantly reduces both acute and chronic rejection in a mouse orthotopic lung transplant model.

In contrast to our prior study, ${ }^{12}$ instead of treating the donor, we devised a combined ex vivo and in vivo gene transfer treatment of the recipient. This proved to be a relatively simple approach that provides a rapid onset and sustained gene expression in the lung allograft after transplantation. By using LV-Luc, we found that this approach was superior to in vivo-only delivery of the transgene, which led to a slower onset and lower peak of expression. In our previous study, as proof-of-principle, we delivered hIL-10 gene to the donor at days 9, 8, and 7 before lung transplant and to the recipient at postoperative days $1,2,3$, and $4 .{ }^{12}$ Obviously, that protocol had no clinical relevance because lung transplantation is not a clinically predictable event. However, we think that using the airway for delivery of gene therapy or immunosuppressive agents ${ }^{25}$ is an attractive option for treatment of acute and chronic rejection. The success of the present study indicates that a combined ex vivo and in vivo lentivirus gene therapy strategy performed at the time of transplant is a superior, more effective strategy that is also a clinically feasible approach.

We showed that combined ex vivo and in vivo gene transfer induces a rapid increase in hIL-10 expression in the allograft. Ex vivo LV-hIL-10 gene delivery resulted in

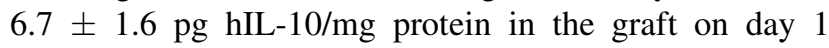
post-transplant. Compared with adenoviral vectors, gene expression from lentiviral vectors is reported to be lower. ${ }^{26}$ Indeed, intra-airway delivery of $4 \times 10^{10}$ plaque-forming units of adenoviral hIL-10 during EVLP in a pig model resulted in $30 \mathrm{pg}$ hIL-10/mg protein after 12 hours. $^{7}$ One of the advantages of using lentiviral vectors, however, is that gene transfer can be repeated because of decreased vector immunogenicity. ${ }^{27}$ In the present study, the hIL-10 expression level in the lung tissue increased to $14.5 \pm 3.4$ $\mathrm{pg} / \mathrm{mg}$ protein at day 7 post-lung transplant after repeated in vivo gene transfers. Adenoviral-delivered hIL-10 could be a therapeutic option to prevent the development of ischemia-reperfusion-induced acute lung injury (PGD) after lung transplant. On the other hand, LV-hIL-10 may be more useful to prevent acute and chronic rejection.

We demonstrated that lentivirus-mediated hIL-10 gene delivery using combined ex vivo and in vivo gene transfer ameliorates AR and airway obliteration at day 28 post-transplant. Okada and colleagues ${ }^{28}$ were unable to reduce AR in rat lungs treated with adenoviral IL-10. They speculated that this may have been related to vector immunogenicity, which might have masked a beneficial effect. Considering our results, we believe that the advantages of lentivirus-mediated gene therapy over adenovirus-mediated therapy include a lower propensity to induce inflammation and longer-term gene expression (at least 1 year; data not shown).

To investigate how IL-10 gene therapy decreases rejection and fibrosis in this model, we assessed endogenous regulatory factors. Lentivirus-mediated delivery of exogenous hIL-10 gene increased mRNA expression of endogenous mIL-10 at day 28 post-transplant. Of note, however, it did not change the expression ratio of Foxp3/CD4 or the number of Foxp $3^{+}$ cells. The ratio of LAG-3 to CD4 expression was 2-fold 

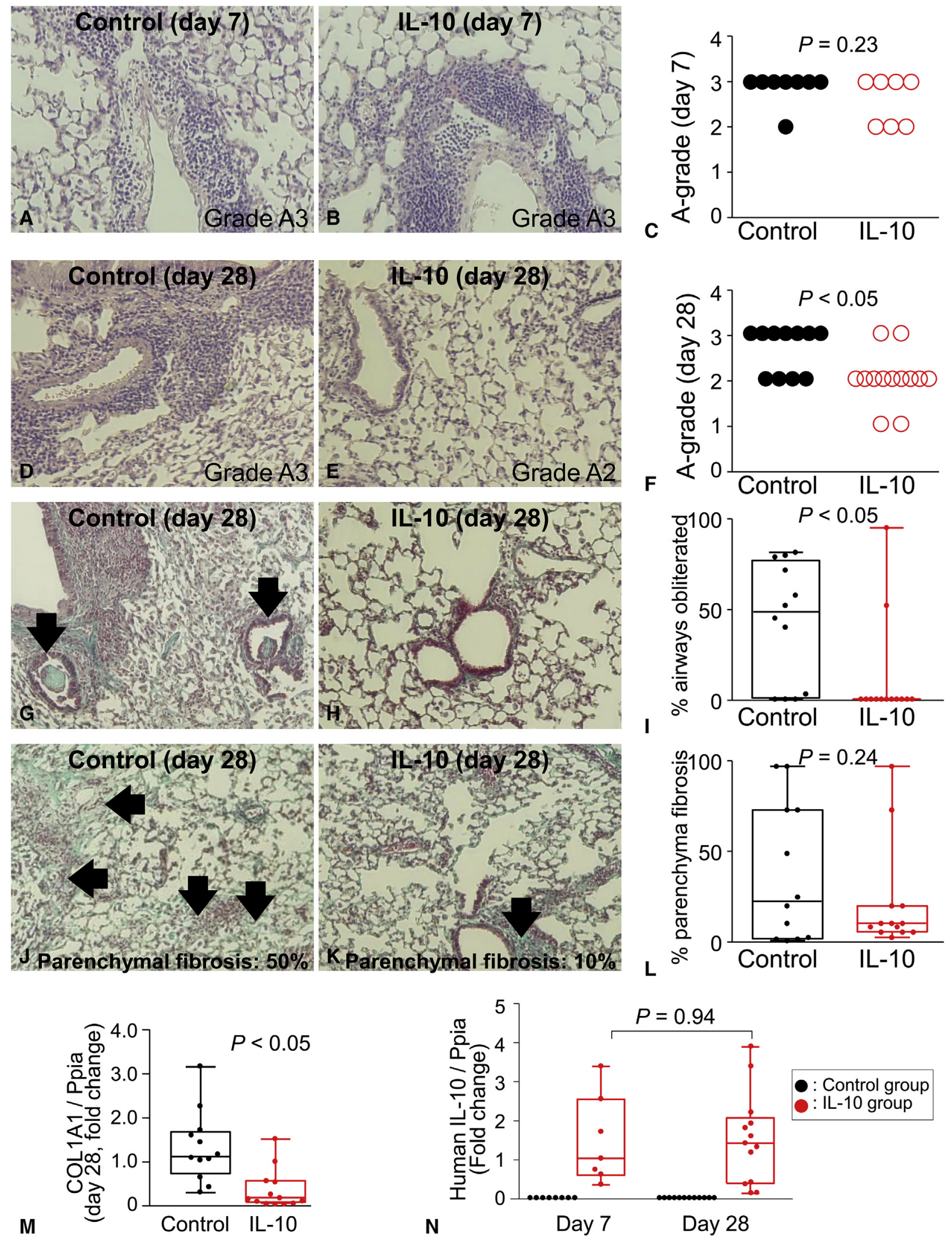

FIGURE 5. Lentivirus-mediated human IL-10 gene delivery using combined ex vivo and in vivo gene transfer ameliorates acute and chronic rejection at day 28. We treated recipient mice in the control group with LV-Luc. In the IL-10 group, recipients received LV-hIL-10. A and B, Representative histology pictures of each group at day 7 post-transplant are shown (hematoxylin-eosin, magnification $20 \times$ ). Mononuclear cells are seen around the blood vessels, airways, and interstitium in both the control and IL-10 groups at day 7. Blinded pathologic scoring of A-grade AR scores (International Society for Heart and Lung Transplantation rejection grade) in both groups. C, AR A grades between the control and IL-10 groups are similar at day 7. Control group, $\mathrm{n}=8$; IL-10 


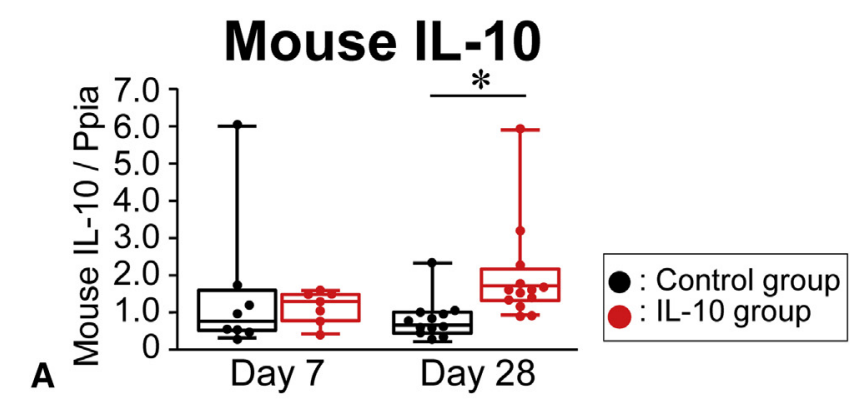

\section{Human IL-10 vs. Mouse IL-10}

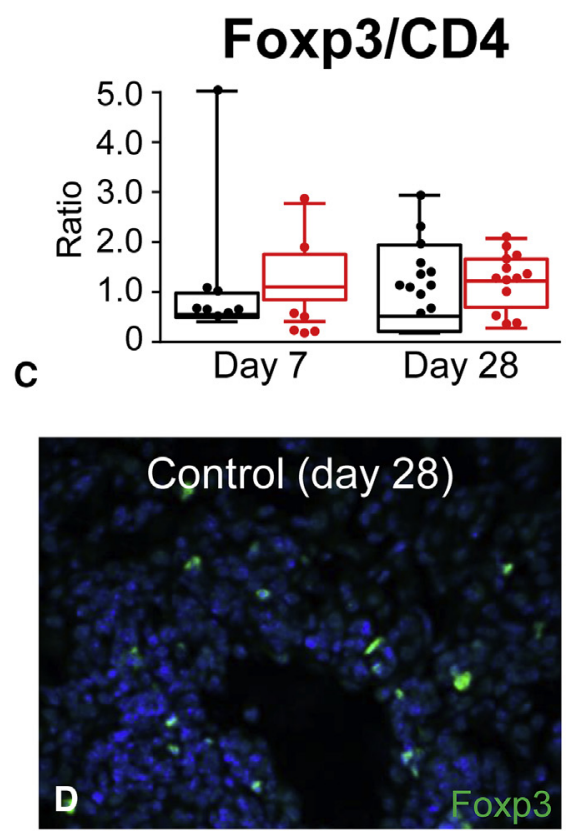

: Control group

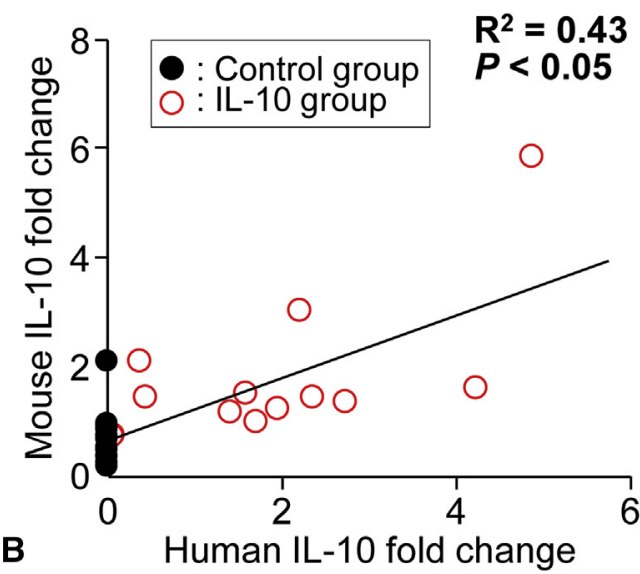

IL-10 group

B Human IL-10 fold change
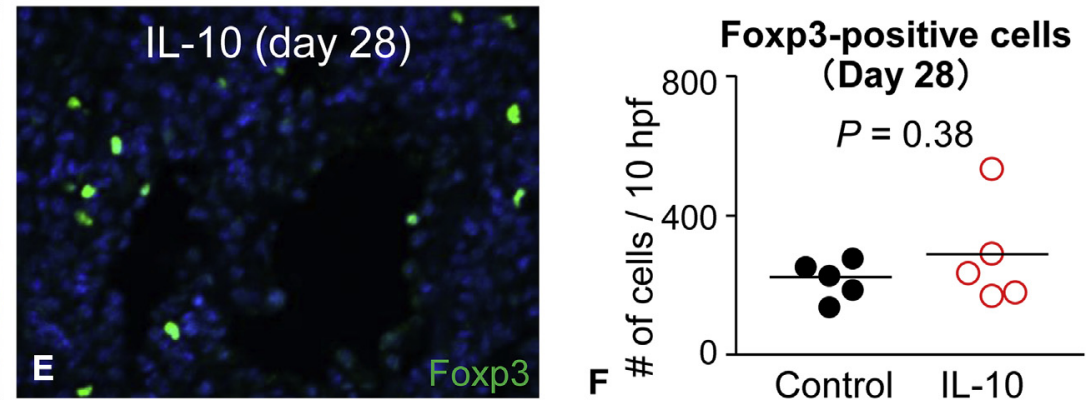

FIGURE 6. Lentivirus-mediated human IL-10 gene delivery using combined ex vivo and in vivo gene transfer increases mRNA expression of mousederived IL-10 at day 28 post-lung transplant. A, LV-hIL-10 increased mRNA expression of mouse IL-10 at day 28 in the lung allograft. B, Induction of mouse IL-10 transcripts in the allograft correlated with the level of human IL-10 expression $\left(\mathrm{R}^{2}=0.43, P<.05\right)$. C, mRNA expression levels of Foxp3 over CD4 at each time point were similar in control and IL-10 groups. Control group, $\mathrm{n}=8$; IL-10 group, $\mathrm{n}=7$ at day 7 . Control group, $\mathrm{n}=12$; IL$10, \mathrm{n}=13$ at day 28 . The upper and lower borders of the box represent the upper and lower quartiles. The middle horizontal line represents the median. The upper and lower whiskers represent the maximum and minimum values of nonoutliers. *Unpaired $t$ test $P<.05$. D and E, Immunofluorescent staining for Foxp3 in representative day 28 lung allografts from the control and IL-10 groups (magnification 20×). F, The number of Foxp3-positive cells per 10 highpower fields in each group is shown. Numbers of Foxp3-positive cells were similar between the control and IL-10 groups at day 28 . $\mathrm{N}=5$ for each group. IL-10, Interleukin-10; Ppia, peptidylprolyl isomerase A; Foxp3, forkhead box P3.

group, $n=7$. D and E, Representative histology pictures of each group at day 28 (hematoxylin-eosin, magnification $20 \times$ ). In the IL-10 group at day 28 , there were fewer infiltrating cells than in the control group. F, A grades in the IL-10 group were significantly lower than in the control group at day 28 (median 2.0 vs 3.0, $P<.05$ ). Control group, $\mathrm{n}=11$; IL-10 group, $\mathrm{n}=13$. (One lung graft from the control group could not be scored because of the severity of the fibrosis.) $\mathrm{G}$ and $\mathrm{H}$, Representative photomicrographs of each group at day 28 post-transplant are shown (Masson's trichrome, magnification $20 \times$ ). Arrows in the control group indicate airway obliterative lesions. I, By blinded semiquantitative pathologic scoring, the percentage of obliterated airways was reduced in the IL-10 group $(10.9 \% \pm 7.7 \%$ vs $40.9 \% \pm 9.3 \%, P<.05)$. Control group, $\mathrm{n}=12$; IL-10 group, $\mathrm{n}=13$. $\mathrm{J}$ and $\mathrm{K}$, Representative photomicrographs of each group at day 28 (Masson's trichrome, magnification 10×). Intense fibrosis in the alveolar parenchyma is shown in the control group. The arrow in the IL-10 group indicates limited fibrosis in the alveolar parenchyma. L, Blinded semiquantitative pathologic assessment of the extent of parenchymal fibrosis (control, $38.3 \% \pm 11.5 \%$ vs IL-10, $21 \% \pm 8.4 \%, P<.05$ ). Control group, $\mathrm{n}=12$; IL-10 group, $\mathrm{n}=13$. M, Analysis of mRNA expression of collagen type I alpha 1 gene in lung grafts at day 28. Data are expressed as mean \pm standard error of the mean. Control group, $\mathrm{n}=12 ; \mathrm{IL}-10 \mathrm{group}, \mathrm{n}=13$. N, mRNA expression of hIL-10 in the graft was measured to confirm gene expression from LV-hIL-10. No mRNA expression of human IL-10 was detected in the lung grafts in the control group at days 7 and 28. All lung grafts in the IL-10 group show mRNA expression levels of hIL-10 at days 7 and 28 . Expression levels are similar at days 7 and 28. The upper and lower whiskers represent the maximum and minimum values of nonoutliers. IL-10, Interleukin-10; COL1A1, collagen type I alpha 1 gene; Ppia, peptidylprolyl isomerase A. 

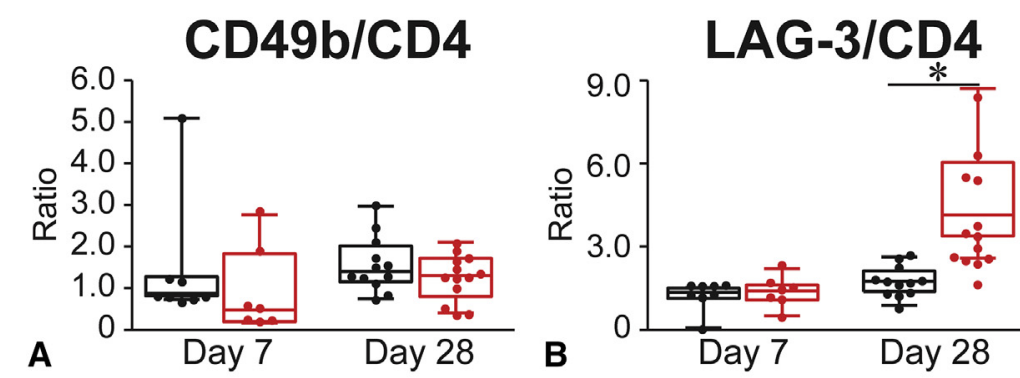

: Control group

IL-10 group

A

Day 7

Day 28

B

\section{LAG-3 vs. mouse IL-10}

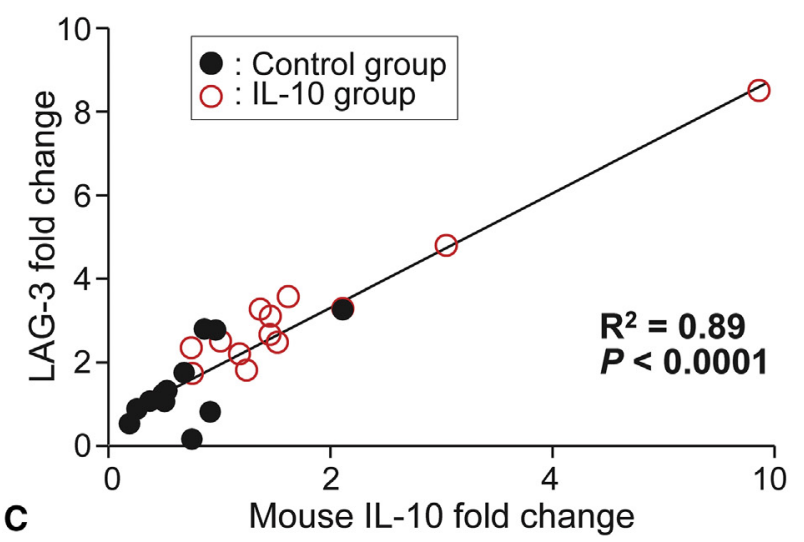

FIGURE 7. Lentivirus-mediated human IL-10 gene delivery using combined ex vivo and in vivo gene transfer increases mRNA expression of LAG-3 at day 28 after lung transplant. A, mRNA expression ratios of CD49b to CD4 at each time point were similar between control and IL-10-treated groups. B, mRNA expression levels of LAG-3 over CD4 were increased at day 28 post-transplant. Control group, $\mathrm{n}=8$; IL-10 group, $\mathrm{n}=7$ at day 7 . Control group, $\mathrm{n}=12$; IL$10, \mathrm{n}=13$ at day 28 . The upper and lower borders of the box represent the upper and lower quartiles. The middle horizontal line represents the median. The upper and lower whiskers represent the maximum and minimum values of nonoutliers. *Unpaired $t$ test $P<.05$. C, mRNA expression levels of LAG-3 correlated with the level of mouse IL-10 expression $\left(\mathrm{R}^{2}=0.89, P<.0001\right) . L A G-3$, Lymphocyte-activation gene 3; IL-10, interleukin-10.

higher in the IL-10 group compared with controls at day 28. These observations suggest that IL-10 may promote $\operatorname{Tr} 1$ cell-mediated local immune regulation, with no effect on Foxp $3^{+}$Treg frequency. This hypothesis is consistent with the observation that IL-10 can differentiate $\mathrm{CD} 4{ }^{+} \mathrm{T}$ cells into $\mathrm{Tr} 1$ cells,${ }^{29}$ whereas transforming growth factor- $\beta$ and IL- 2 are required for induced Foxp $3^{+}$Treg generation. ${ }^{30}$ Although our data suggest that IL-10-induced Tr1 cells may underlie the inhibition of lung AR by IL-10 gene therapy, more refined single cell analyses and functional studies will be required to establish the specific regulatory cell populations involved.

\section{CONCLUSIONS}

Lentiviral hIL-10 gene therapy delivered at the time of lung transplant with a combined ex vivo and in vivo technique provides rapid and durable upregulation of IL-10 expression that inhibits acute and chronic rejection in a mouse lung transplant model. These beneficial effects were accompanied by upregulation of endogenous mIL-10 and LAG-3, suggesting the involvement of $\operatorname{Tr} 1$ regulatory cells.

\section{Conflict of Interest Statement}

Drs Oishi, Juvet, and Keshavjee report grants from Cystic Fibrosis Canada and the Canadian Institutes for Health Research during the conduct of this study. All other authors have nothing to disclose with regard to commercial support.

The authors thank Jerome Valero for editing the manuscript and Paul Chartrand for organizing and facilitating lung tissue sample processing. We also acknowledge Reza Mirza for assistance with ELISA.

\section{References}

1. Yusen RD, Edwards LB, Kucheryavaya AY, Benden C, Dipchand AI, Dobbels F, et al. The registry of the International Society for Heart and Lung Transplantation: thirty-first adult lung and heart-lung transplant report-2014; focus theme: retransplantation. J Heart Lung Transplant. 2014;33:1009-24.

2. Whitson BA, Prekker ME, Herrington CS, Whelan TP, Radosevich DM, Hertz MI, et al. Primary graft dysfunction and long-term pulmonary function after lung transplantation. J Heart Lung Transplant. 2007;26:1004-11.

3. Fischer S, Liu M, Alexandra A, de Perrot M, Ho M, Cardella JA, et al. In vivo transtracheal adenovirus-mediated transfer of human interleukin-10 gene to donor lungs ameliorates ischemia-reperfusion injury and improves early posttransplant graft function in the rat. Hum Gene Ther. 2001;12:1513-26.

4. de Perrot M, Fischer S, Liu M, Imai Y, Martins S, Sakiyama S, et al. Impact of human interleukin-10 on vector-induced inflammation and early graft function in rat lung transplantation. Am J Respir Cell Mol Biol. 2003;28:616-25. 
5. Fischer S, de Perrot M, Liu M, MacLean AA, Cardella JA, Imai Y, et al. Interleukin 10 gene transfection of donor lungs ameliorates posttransplant cell death by a switch from cellular necrosis to apoptosis. J Thorac Cardiovasc Surg. 2003;126:1174-80.

6. Martins S, de Perrot M, Imai Y, Yamane M, Quadri SM, Segall L, et al. Transbronchial administration of adenoviral-mediated interleukin-10 gene to the donor improves function in a pig lung transplant model. Gene Ther. 2004; 11:1786-96.

7. Cypel M, Liu M, Rubacha M, Yeung JC, Hirayama S, Anraku M, et al. Functional repair of human donor lungs by IL-10 gene therapy. Sci Transl Med. 2009;1:4ra9.

8. Yeung JC, Wagnetz D, Cypel M, Rubacha M, Koike T, Chun YM, et al. Ex vivo adenoviral vector gene delivery results in decreased vector-associated inflammation pre- and post-lung transplantation in the pig. Mol Ther. 2012;20: 1204-11.

9. Machuca TN, Cypel M, Bonato R, Yeung JC, Chun YM, Juvet S, et al. Safety and efficacy of ex vivo donor lung adenoviral IL-10 gene therapy in a large animal lung transplant survival model. Hum Gene Ther. 2017;28:757-65.

10. Jaffe HA, Danel C, Longenecker G, Metzger M, Setoguchi Y, Rosenfeld MA, et al. Adenovirus-mediated in vivo gene transfer and expression in normal rat liver. Nat Genet. 1992;1:372-8.

11. Hirayama S, Sato M, Liu M, Loisel-Meyer S, Yeung JC, Wagnetz D, et al. Local long-term expression of lentivirally delivered IL-10 in the lung attenuates obliteration of intrapulmonary allograft airways. Hum Gene Ther. 2011;22: 1453-60.

12. Hirayama S, Sato M, Loisel-Meyer S, Matsuda Y, Oishi H, Guan Z, et al. Lentivirus IL-10 gene therapy down-regulates IL-17 and attenuates mouse orthotopic lung allograft rejection. Am J Transplant. 2013;13:1586-93.

13. Shoji F, Yonemitsu Y, Okano S, Yoshino I, Nakagawa K, Nakashima Y, et al. Airway-directed gene transfer of interleukin-10 using recombinant Sendai virus effectively prevents post-transplant fibrous airway obliteration in mice. Gene Ther. 2003;10:213-8.

14. Okazaki M, Krupnick AS, Kornfeld CG, Lai JM, Ritter JH, Richardson SB, et al. A mouse model of orthotopic vascularized aerated lung transplantation. Am J Transplant. 2007;7:1672-9.

15. Bradford MM. A rapid and sensitive method for the quantitation of microgram quantities of protein utilizing the principle of protein-dye binding. Anal Biochem. 1976;72:248-54.

16. Stewart S, Fishbein MC, Snell GI, Berry GJ, Boehler A, Burke MM, et al. Revision of the 1996 working formulation for the standardization of nomenclature in the diagnosis of lung rejection. J Heart Lung Transplant. 2007;26:1229-42.

17. Sato M, Liu M, Anraku M, Ogura T, D’Cruz G, Alman BA, et al. Allograft airway fibrosis in the pulmonary milieu: a disorder of tissue remodeling. Am J Transplant. 2008;8:517-28.
18. Ofek E, Sato M, Saito T, Wagnetz U, Roberts HC, Chaparro C, et al Restrictive allograft syndrome post lung transplantation is characterized by pleuroparenchymal fibroelastosis. Mod Pathol. 2013;26:350-6.

19. Pontoux C, Banz A, Papiernik M. Natural CD4 CD25(+) regulatory T cells control the burst of superantigen-induced cytokine production: the role of IL-10. Int Immunol. 2002;14:233-9.

20. Mizoguchi A, Mizoguchi E, Takedatsu H, Blumberg RS, Bhan AK. Chronic intestinal inflammatory condition generates IL-10-producing regulatory B cell subset characterized by CD1d upregulation. Immunity. 2002;16:219-30.

21. Roncarolo M-G, Battaglia M. Regulatory T-cell immunotherapy for tolerance to self antigens and alloantigens in humans. Nat Rev Immunol. 2007;7: 585-98.

22. Arase H, Saito T, Phillips JH, Lanier LL, Alerts E. Cutting edge: the mouse NK cell-associated antigen recognized by DX5 monoclonal antibody is CD49b ( $\alpha 2$ Integrin, very late antigen-2). J Immunol. 2016;167:1141-4.

23. $\mathrm{Xu} \mathrm{J}$, Clark RAF. Extracellular matrix alters PDGF regulation of fibroblast integrins. J Cell Biol. 1996;132:239-49.

24. Sierro S, Romero P, Speiser DE. The CD4-like molecule LAG-3, biology and therapeutic applications. Expert Opin Ther Targets. 2011;15:91-101.

25. Das NA, Peters JI, Simmons JD, Wang Y, O'Donnell KP, DeArmond DT, et al The efficacy of inhaled nanoparticle tacrolimus in preventing rejection in an orthotopic rat lung transplant model. J Thorac Cardiovasc Surg. 2017;154: 2144-51.e1.

26. Merentie M, Lottonen-Raikaslehto L, Parviainen V, Huusko J, Pikkarainen S, Mendel M, et al. Efficacy and safety of myocardial gene transfer of adenovirus, adeno-associated virus and lentivirus vectors in mouse heart. Gene Ther. 2015; 23:1-10.

27. Griesenbach U, Alton EW. Progress in gene and cell therapy for cystic fibrosis lung disease. Curr Pharm Des. 2012;18:642-62.

28. Okada Y, Zuo XJ, Toyoda M, Marchevsky A, Matloff JM, Oishi H, et al Adenovirus mediated IL-10 gene transfer to the airway of the rat lung for prevention of lung allograft rejection. Transpl Immunol. 2006;16:95-8.

29. Groux H, Garra A, Bigler M, Rouleau M, Antonenko S, de Vries JE, et al. A CD4+ T-cell subset inhibits antigen-specific T-cell responses and prevents colitis. Nature. 1997;389:737-42.

30. Chen W, Jin W, Hardegen N, Lei KJ, Li L, Marinos N, et al. Conversion of peripheral $\mathrm{CD} 4+\mathrm{CD} 25$ - naive $\mathrm{T}$ cells to $\mathrm{CD} 4+\mathrm{CD} 25+$ regulatory $\mathrm{T}$ cells by TGF- $\beta$ induction of transcription factor Foxp3. J Exp Med. 2003;198: 1875-86.

Key Words: lung transplantation, interleukin-10, lentivirus, acute rejection, chronic rejection 